\title{
基于改进潜能模型的城市医疗设施空间可达性 以上海市杨浦区为例
}

\author{
程敏”, 连月娇
}

(上海大学管理学院, 上海 200444)

\begin{abstract}
摘 要:引人人口规模因子和医疗设施等级规模影响系数改进潜能模型, 基于 GIS 空间分析技术, 以上海杨浦区为 例对其医疗设施空间可达性进行研究。结果表明:杨浦区医疗设施空间可达性分布不均, 大桥街道、定海路街道及 殷行街道部分区域医疗设施空间可达性较高; 中部区域一些街道存在同等级医院位置密集、医疗资源相对集中的 情况; 杨浦区三级医院的居民就医空间可达性整体优于二级、一级医院。改进的潜能模型综合考虑了居民点人口 数量、医疗设施与居民点之间的出行阻抗、以及医疗设施的等级规模对居民就医选择的影响, 能更合理地评价医疗 设施的可达性。研究结果可为城市医疗设施科学规划与决策提供依据。
\end{abstract}

关 键 词: 医疗设施; 空间可达性; 改进潜能模型; GIS; 杨浦区; 上海

\section{1 引言}

医疗服务是公众的基本需求, 与公众的身体健 康密切相关, 医疗服务设施布局的合理性与可达性 则关系到公众健康权能否得到有效保障。医疗设 施的可达性是指某区域范围内居民通过各种方式 获得医疗服务或到达医疗设施的便捷程度, 以到达 医疗设施的能力(多指花费的时间或距离)或可获得 的服务的数量和质量来衡量。优化医疗设施布局、 提高医疗设施的可达性是实现区域医疗服务均等 化的有效途径。

空间可达性测度是对公共服务设施空间配置 是否合理进行度量的有效方法之一, 相比城市规划 评价公共设施服务能力的指标(如千人床位数), 其 能更直观、全面地反映设施空间分布的均衡性, 因 此在教育、文体、医疗等公共服务设施配置中广泛 应用。空间可达性测度常用的方法有: 比例模型法 (孔云峰等, 2008)、最小距离模型(Fan et al, 2017; 曾 文等, 2017)、两步移动搜索法(邓丽等, 2015; 钟少颖 等, 2016)、潜能模型(Gharani et al, 2015; Wu et al,
2017; 汤鹏飞等, 2017)和交通网络中心性测度法 (Porta et al, 2012; 陈晨等, 2014)等。与其他可达性 模型相比, 潜能模型能综合考虑居民需求和空间阻 隔(时间、距离等),并能精确反映较小的空间尺度研 究单元内居民获得设施资源的情况。目前, 潜能模 型被应用于医疗服务可达性评价。由于传统潜能 模型忽略了人口数量及不同设施规模等级等关键 因素, 因此,一些学者对传统潜能模型进行了改进, 在模型中考虑了人口规模、医疗设施等级对居民就 医选择行为的影响或其他现实因素 (吴建军等, 2008; 宋正娜等, 2009, 2010; 熊娟等, 2012; Yang et al, 2016)。

现有研究在选取医疗设施空间可达性模型的 研究单元时,大多以县(区)(Cheng et al, 2016; Pan et al, 2016)、街道(钟少颖等, 2016; 丁愫等, 2017; 佟圣 楠等, 2017)为研究单元, 较少以居住区为研究单 元。事实上, 选取较小的研究单元, 更能有效地反 映区域内居民医疗设施空间可达性的差异,准确地 判断最缺医的基层单元。因此, 医疗设施空间可达 性模型的研究单元选取有待进一步细化。

收稿日期: 2017-07-19; 修订日期: 2017-11-24。

基金项目: 上海市哲学社会科学规划项目(2016BGL008); 上海市浦江人才计划项目(15PJC047) [Foundation: Shanghai Philosophy and Social Science Program, No.2016BGL008; Shanghai Pujiang Program, No.15PJC047]。

作者简介: 程敏,女, 湖北荆门人, 博士, 副教授, 主要研究方向为公共设施配置研究, E-mail: chengmin@yeah.net。 
基于 GIS 技术的空间可达性分析可弥补传统 规划对于设施空间位置缺乏考虑的不足,一些学者 将 GIS 技术应用于医疗设施可达性的研究。如 Kalogirou等(2006)基于 GIS 技术, 采用加权可达性 模型, 分析了爱尔兰部分地区的医疗设施可达性; 林康等(2009)基于 GIS 技术, 用时间成本反映空间 距离, 研究了江苏省仪征市医院空间布局; 胡瑞山 等(2012)基于江苏省东海县的道路拓扑网络,利用 两步移动搜索法计算东海县村级居民点医疗空间 可达性, 确定东海县的缺医地区; 孙瑜康等(2015)综 合采用GIS、问卷调查、出行路线图分析等多种技术 对江西德兴市医疗设施配置进行评价, 以反映服务 设施的空间可达性、服务质量、居民的需求与选 择。虽然, 基于 GIS 技术的医疗设施可达性研究取 得了一定进展, 但仍存在一定的局限性, 部分研究 尽管考虑了不同等级道路的不同时速和实际的道 路距离, 但对于发达城市来说, 尚未考虑地面交通 和轨道交通构成的综合交通网络因素对可达性的 影响。

本文拟以上海市杨浦区为例, 对其医疗设施空 间可达性进行研究。研究以居住区为研究单元, 针 对医疗设施的实际需求, 纳人人口规模和医疗设施 等级规模 2 个因素, 对传统潜能模型进行改进和修 正, 并在运用GIS 技术进行可达性可视化分析时, 结 合上海交通特点, 综合考虑地面交通和轨道交通相 结合而形成的综合交通网络的影响, 以便更为科学 地衡量医疗设施的空间分布情况及其合理性, 从而 为政府进一步优化医疗设施配置提供参考。

\section{2 研究方法}

\section{1 潜能模型}

潜能模型是借助物理万有引力定律研究经济、 社会空间相互作用的一种模型。引力模型来源于 牛顿重力模型, 由于社会现象具有复杂性, 原始的 引力模型与实际状况还存在较大偏差。因此, 法国 学者 Louis Lagarange 在牛顿万有引力定律的基础 上提出了万有引力潜能的概念, 即一个物体对另一 个物体产生的能。这一概念后来被引人人文地理 和区域经济研究中, 并发展为研究空间相互作用的 潜能模型。潜能模型基本表达为:

$$
A_{i}=\sum_{j=1}^{n} A_{i j}=\sum_{j=1}^{n} \frac{M_{j}}{D_{i j}^{\beta}}
$$

式中: $A_{i j}$ 表示出行摩擦系数为 $\beta$ 时,物体 $j$ 对物体 $i$ 产生的潜能; $M_{j}$ 表示 $j$ 点的活动规模; $D_{i j}^{\beta}$ 表示出行 摩擦系数为 $\beta$ 时 $i$ 点到 $j$ 点的出行阻抗因子(距离或 时间,本文以时间表示); $A_{i}$ 表示系统中所有物体对 $i$ 点所产生的潜能集合。由式(1)可知,出行阻抗因子 降低, $A_{i}$ 相应增加, $A_{i}$ 越大, 说明空间可达性越好。 关于出行摩擦系数 $\beta$ 的取值, 学界认为不同设施服 务类型和人群特征的 $\beta$ 取值不同,且 $\beta$ 取值会影响 度量的准确度。

此后, 学界将潜能模型中的变量进一步细化定 义,并用于度量各类设施的空间可达性。具体到医 疗设施领域, 该模型的含义为: 在考虑设施服务能 力和出行阻抗时, 研究区域内某一居民点 $i$ 到所有 医疗设施的可达性 $A_{i}$, 即研究区域内各医疗设施对 居民点 $i$ 的吸引力的累计值。然而, 基本潜能模型 公式仅仅涉及设施服务能力 $M_{j}$ (本文以病床数表示) 和出行阻抗因素 $D_{i j}^{\beta}$, 未考虑人口因素, 即同一设施 周围服务人口之间对设施资源的竞争。当设施服 务能力相同、出行阻抗相同但设施服务辐射人口数 量有差异时,式(1)无法明显区分两点实际空间可达 性的差异。

\section{2 潜能模型的改进}

Weibull(1976)考虑消费者之间的供需竞争, 将 人口规模因子 $V_{j}$ 纳人基本公式, 进一步完善了潜能 模型, 公式为:

$$
A_{i}=\sum_{j=1}^{n} \frac{M_{j}}{D_{i j}^{\beta} V_{j}} \text {, 其中 } V_{j}=\sum_{k=1}^{m} \frac{P_{k}}{D_{k j}^{\beta}}
$$

式中: $V_{j}$ 为人口规模影响因子; $P_{k}$ 表示居民点 $k$ 的人 口数; $n$ 和 $m$ 分别表示医疗设施和居民点的数量; $D_{k j}^{\beta}$ 表示出行摩擦系数为 $\beta$ 时, 居民点 $k$ 与医疗设施点 $j$ 的 出行阻抗因子(距离或时间,本文以时间表示)。以上 模型的改进考虑了各医疗设施附近的人口分布, 反 映居民到同一医疗设施就医造成对有限医疗资源 的竞争, 从而负面影响空间可达性,但是并未考虑 不同等级医疗设施规模对居民就医选择的影响。

2015 年 3 月国务院办公厅印发的《全国医疗卫 生服务体系规划纲要(2015-2020年)》, 以及2017年 国家卫生和计划生育委员会出台的《医疗机构管理 条例实施细则》等文件中对中国医疗服务体系做出 了明确的分级规定。2011年出台的《上海市医疗机 构管理办法》及《上海市区域卫生规划(2011-2020 年)》也对各级医疗设施的规模和服务范围进行了 
界定和说明。医疗设施的规模大小及医疗服务水 平的高低与其影响范围有直接关系。医疗设施的 规模越大, 医疗水平越高, 该医疗设施对居民的影 响范围也越大, 即医疗设施在吸引其周边的居民之 外还能辐射到距离更远的居民。因此, 在计算医疗 设施可达性时还应考虑到医疗设施规模对居民就 医选择的影响。本文在式(2)所示的改进潜能模型 中通过加人医疗设施等级规模影响系数 $S_{i j}$ 来体现 这一影响。公式如下:

$$
A_{i}=\sum_{j=1}^{n} \frac{S_{i j} M_{j}}{D_{i j}^{\beta} V_{j}} \text {, 其中 } V_{j}=\sum_{k=1}^{m} \frac{S_{k j} P_{k}}{D_{k j}^{\beta}}, S_{i j}=1-\left(\frac{D_{i j}}{D_{j}}\right)^{\beta}
$$

式中: $P_{k}$ 表示居民点 $k$ 的人口数; $S_{i j}$ 表示医疗设施 $j$ 对居民点 $i$ 的等级规模影响系数; $S_{k j}$ 表示医疗设施 点 $j$ 的等级规模对居民点 $k$ 就医行为的影响; $D_{i j}$ 表 示居民点 $i$ 与医疗设施点 $j$ 的时间成本; $D_{j}$ 表示极限 出行时间, 当居民点到设施点的出行时间超过极限 出行时间, 即 $\frac{D_{i j}}{D_{j}} \geqslant 1$ 时, $S_{i j} \leqslant 0$, 即居民不选择该点 就医。式(3)所示的改进潜能模型综合考虑了居民 点人口数量、医疗设施服务能力、医疗设施与居民 点之间的出行阻抗、不同等级规模医疗设施对居民 就医行为的影响等因素, 相对传统潜能模型而言能 更好地反映实际情况。

\section{3 可达性测算与分析}

\section{1 研究区域与数据准备}

本文以上海市杨浦区为例, 基于 ArcGIS 软件 平台, 度量其医疗设施空间可达性, 评价杨浦区医 疗设施现状。杨浦区位于上海市中心城区东北部, 中心经纬度为 $31^{\circ} 27^{\prime} \mathrm{N} 、 121^{\circ} 52^{\prime} \mathrm{E}$, 地处黄浦江下游 西北岸, 与浦东新区隔江相望, 西临虹口区, 北与宝 山区接壤。全区面积 $60.61 \mathrm{~km}^{2}, 2016$ 年人口 130.94 万人。辖区内共有 12 个街道(镇), 分别为: 定海路 街道、平凉路街道、江浦路街道、四平路街道、控江路 街道、长白新村街道、延吉新村街道、殷行街道、大桥 街道、五角场街道、新江湾城街道、五角场镇。区境 是长江三角洲前沿冲积平原, 区内地势平坦, 路网 密布, 地形单一。研究区域概况如图 1 所示。

医疗设施系统具有典型的层级性特征, 不同等 级规模的医院对于居民就医需求的吸引力也有所 不同。《上海市医疗机构管理办法》将医院按其功

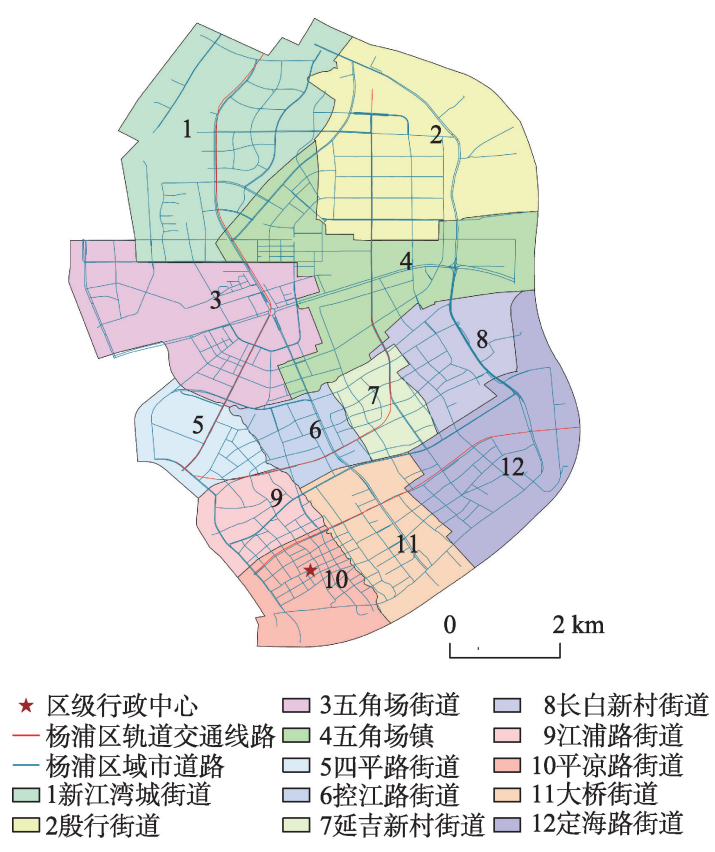

图 1 研究区域概况

Fig.1 Overview of the study area

能、任务不同, 由低到高划分为一、二、三级。《上海 市区域卫生规划(2011-2020年)》中则明确规定一级 医院主要承担一般常见病、多发病的全科诊疗及分 诊、基本公共卫生等服务; 二级医院为区域内居民 提供常见病专科门诊、急诊、重症医疗、手术和住院 服务; 三级医院承担重疑难病症的诊治任务和相应 的公共卫生职能。三级医院医疗设施规模较大, 技 术力量雄厚, 对疑难杂症的治疗水平更高, 因此除 满足周边居民的需求外也会吸引距离较远的居 民。一级或二级医院在医护人员数量、医疗救治水 平方面相对较弱, 但设置数量上比三级医院多, 能满 足居民轻微病痛治疗需求, 但不会吸引较远地区的 居民。为便于研究, 本文选取杨浦区的公立一、二、 三级医院进行研究。杨浦区公立医院共 27 个, 其中 三级医院 6 个, 二级医院 10 个, 一级医院 11 个, 医疗 设施分布情况如图 2 所示。本文数据主要来源于 《杨浦区统计年鉴》及上海市卫计委、各医院官网。

杨浦区下设 12 个街道(镇)、305个居委会, 居民 点分布情况如图 3 所示。为了有效、准确地描述居 民医疗设施空间可达性的差异, 本文以居住区为研 究单元。由于缺少街道(镇)下一级的面图层数据, 无法以几何质心作为人口重心, 因此, 借鉴已有研 究(宋正娜等, 2009, 2010; 陶卓霖等, 2014), 以居住 区的行政中心一居委会代替人口重心, 并选取了 杨浦区部分居委会(如上理居委会、民治路居委会 


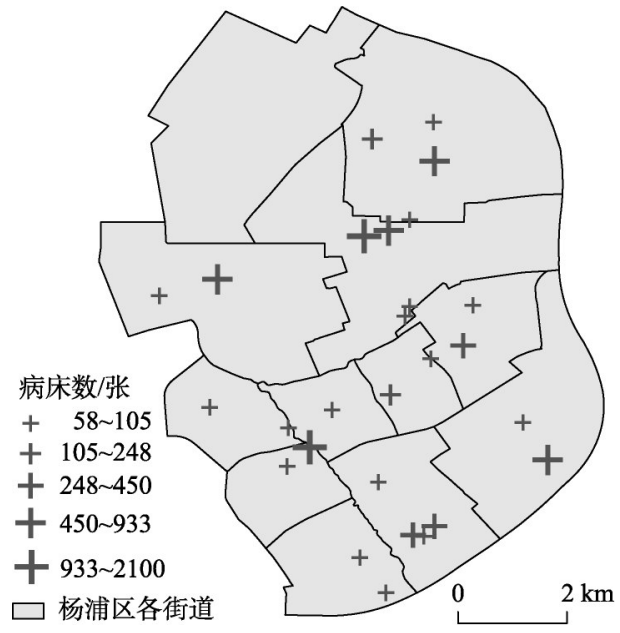

图 2 杨浦区医疗设施分布

Fig.2 Distribution of medical facilities in Yangpu District

等)对该取值方法的可靠性进行验证,结果表明计 算得到的居住区人口重心坐标与居委会的实际地 理坐标误差较小,表明该取值方法可行。

\section{2 空间可达性计算}

本文基于 ArcGIS 中的 Network 分析模块, 以设 施点、居民点及路网(包含公共交通网络)为基础, 计 算居民最短出行时间。居民就医出行时间的计算， 考虑了地面交通和轨道交通相结合的情况。

关于地面交通,一些学者(胡瑞山等, 2012; 孟 田田等, 2017)在研究中采用《公路技术标准》 (JIGB01-2003)、《城市道路工程设计规范》(CJJ372012)中规定的各级道路行车速度计算通行时间。 但对于上海而言,城市交通存在拥堵的情况，实际 的道路运行速度与上述规范的数值差异较大, 因 此,本文选取城市高峰时段道路的平均运行速度为 道路赋值，近似计算日常生活中实际的出行时间。 不同等级道路速度采用上海市城乡建设和交通发 展研究院、上海城市综合交通规划研究所发布的 《2016上海市综合交通运行年报》中的数据, 如表 1 所示。

对于轨道交通而言, 通常地铁的设计时速达 120 180 km/h, 但在实际运行过程中, 考虑到线路 条件(弯道、坡道)、站点设置(站间距)、列车停站时 间(客流大小)等因素, 运行速度一般不超过 $80 \mathrm{~km} / \mathrm{h}$

表 1 中心城区各级道路速度设置

Tab.1 Speed limit for different types of road in the city center of Shanghai

\begin{tabular}{cccc}
\hline 道路类型 & 快速路 & 主干道 & 次干道、支路及以下 \\
\hline 速度 $/(\mathrm{km} / \mathrm{h})$ & 38.6 & 14.9 & 17.5 \\
\hline
\end{tabular}

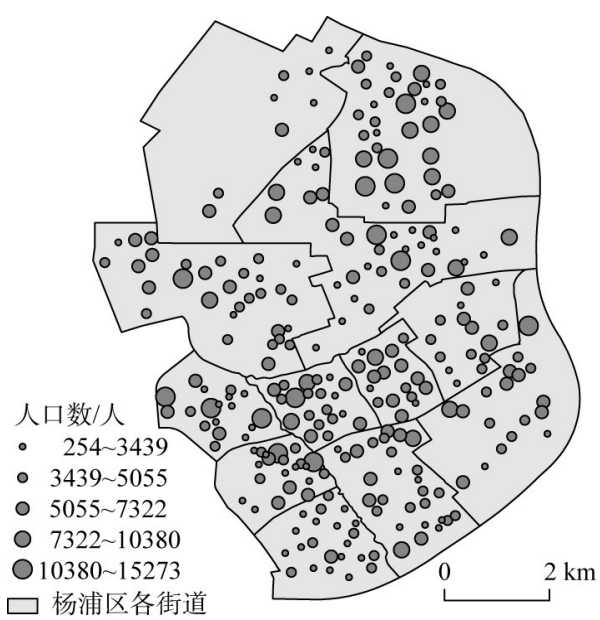

图 3 杨浦区居民点分布

Fig.3 Distribution of residential area in Yangpu District

(邓羽等, 2012)。依据上海测绘院与上海地铁官方 网站提供的地铁线路里程和运行时间,计算得到每 一条地铁线路(杨浦区主要涉及 $8 、 10 、 12$ 号线)的运 行速度,如表 2 所示。

一般来说, 居民前往某个公共设施所能承受的 出行时间有一个阈值(即极限出行时间 $D_{j}$ ), 本文分 别对一、二、三级医院的 $D_{i}$ 进行了设定。参考《上海 市城市 15 分钟生活圈规划总则(试行)》、《上海城市 总体规划(2016-2040)》中关于构建 15 分钟生活圈 的说明,以及上海“1560 就医圈”医疗服务网络构建 的实际情况,将一级医院的 $D_{j}$ 设为 15 分钟; 通过实 地走访社区抽样调查居民的就医出行时间,综合不 同社区的结果 , $40.6 \%$ 居民认为前往二级医院就医 的时间为 20 分钟以内较为合理, 53.4\%的居民认为 前往二级医院就医的时间为 20 30 分钟较为合理, 因此将二级医院的 $D_{j}$ 设为 30 分钟; 由于三级医院医 疗资源丰富, 医疗技术先进,且在重疑难病症的诊 治方面具有优势, 考虑居民对三级医院就医的实际 需求, 将其 $D_{i}$ 设为 $+\infty$ 。

\section{3 结果分析}

3.3.1 基于比例模型的初步分析

根据国家住建部发布的《城市居住区规划设计

表 2 各地铁线路运行情况表

Tab.2 Speed of different metro lines

\begin{tabular}{rcccc}
\hline 线路名 & $\begin{array}{c}\text { 线路全长 } \\
/ \mathrm{km}\end{array}$ & $\begin{array}{c}\text { 车站数 } \\
/ \text { 座 }\end{array}$ & $\begin{array}{c}\text { 全程时间 } \\
/ \mathrm{min}\end{array}$ & $\begin{array}{c}\text { 平均速度 } \\
/(\mathrm{km} / \mathrm{h})\end{array}$ \\
\hline 8 号线 & 37.7 & 30 & 65 & 34.80 \\
10 号线 & 30.5 & 28 & 64 & 28.59 \\
12 号线 & 37.4 & 32 & 77 & 29.10 \\
\hline
\end{tabular}


规范》(GB50180-93)和各省市城市公共服务设施规 划标准, 各类公共服务设施的配置大多采用千人指 标, 即特定区域内资源总量与人口数量之比表示。 上海市 2016 年卫生计生数据显示, 上海市每千人享 有病床数为 8.9 张。本文以杨浦区 3 个级别的医疗 设施病床数为资源总量, 进行比例模型分析, 得到 杨浦区各街道千人病床密度分布, 如图 4 所示。在 12 个街道(镇)中, 五角场镇和江浦路街道情况较好, 每千人病床数高于上海人均标准; 五角场街道、大 桥街道、定海路街道、殷行街道次之; 四平路街道、 延吉新村街道、长白新村街道、控江路街道、平凉路 街道和新江湾城街道资源数量分布少于其他街 道。可见, 基于千人指标的分析能大致判断出区域 内各街道(镇)的居民拥有医疗设施的情况, 但未能 考虑到设施的服务质量、居民人口分布、居民点与 设施点间的距离、居民的需求和选择。因此, 比例 模型仅能初步了解区域的设施分布情况, 准确度较 低且有一定局限性。

\subsection{2 基于改进潜能模型和 GIS 的可达性评价}

(1) 出行摩擦系数 $\beta$ 值的选取

关于 $\beta$ 的取值, 较为理想的做法是依据设施的 实际使用情况确定, 可通过与设施不同距离下的使 用人数回归得到。例如, Reggiani 等(2011)基于 2003 年和 2007 年德国 439 个地区居民日常通勤流 量数据, 采用回归分析计算得到不同距离衰减函数 下的 $\beta$ 值。然而, 这种方法往往需要大量的数据、 成本较高、实现较为困难。因此, 实际研究中, 多采

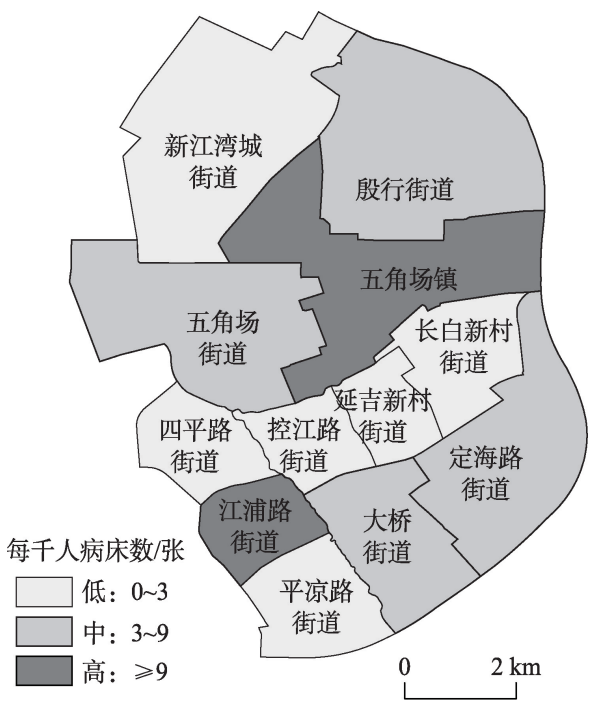

图 4 比例模型下杨浦区各街道(镇)千人病床数情况

Fig.4 The number of hospital beds per 1000 persons in different subdistricts of Yangpu District
用对参数的多种取值进行多情景分析的方法(陶卓 霖等, 2017)。Peeters 等(2000)在总结前人研究的基 础上, 指出出行摩擦系数 $\beta$ 的取值集中在 $[0.9$,

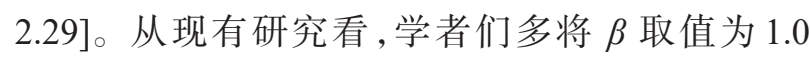
(Ortega et al, 2012; 丁秋贤等, 2016)、1.5(Suárez et al, 2012; Siegel et al, 2016)、1.8(Reggiani et al, 2011) 和 2.0(如 Yang et al, 2016; 汤鹏飞等, 2017)。本文将 这 4 个值分别代人式(3)进行计算, 探讨不同 $\beta$ 取值 对医疗设施空间可达性的影响。4 种情况的描述性 统计分析结果如表 3 所示, 由表可看出, 随着 $\beta$ 值 的增大, 可达性的最大值增大, 最小值减小, 标准差 增大, 可达性结果的分布逐渐呈现出 “发散” 的特 点, 说明可达性评价结果对 $\beta$ 取值的敏感程度较 高, 需选择合适的 $\beta$ 值进行计算。通过选取社区样 本, 走访调查居民的日常平均出行时间, 与上述 4 种 情况下计算的出行时间进行对比, 发现当 $\beta=1.8$ 时, 得到的各点的出行时间与实际较为吻合。因 此, 选取 $\beta=1.8$ 对杨浦区医疗设施空间可达性进行 计算和分析。

\section{(2) 基于 GIS 的插值法选择}

为描绘测量点及其周围未知区域的医疗设施 空间可达性,需对采用改进潜能模型计算得到的居 民点的医疗设施空间可达性值进行插值分析。本文 对比了几种常规插值方法和地统计学方法, 以期选 择具有较好插值效果的方法,计算结果如表4所示。

一般来说, 误差均值 $M E A N$ 和均方根误差

表3 杨浦区各居民点医疗设施可达性结果描述性统计

Tab.3 Descriptive statistics of spatial accessibility of medical facilities in Yangpu District

\begin{tabular}{crrrr}
\hline 统计指标 & $\beta=1.0$ & $\beta=1.5$ & $\beta=1.8$ & $\beta=2.0$ \\
\hline 最大值 & 29.09 & 37.62 & 43.79 & 48.52 \\
最小值 & 2.61 & 1.83 & 1.44 & 1.22 \\
标准差 & 3.34 & 4.43 & 5.38 & 6.21 \\
\hline
\end{tabular}

表4 不同插值方法的结果比较

Tab.4 Comparison of the results calculated by different interpolation methods

\begin{tabular}{lllrc}
\hline & \multicolumn{1}{c}{ 插值方法 } & 参数模型 & $M E A N$ & $R M S E$ \\
\hline 常规 & 反距离加权插值法 & 指数为 2 & -0.079 & 4.581 \\
方法 & 全局多项式插值法 & 指数为 3 & 0.021 & 5.181 \\
& 局部多项式插值法 & 指数为 3 & -0.121 & 5.654 \\
& 径向基函数插值法 & 张力样条函数 & 0.033 & 4.465 \\
地统计 & 经验贝叶斯克里金 & 无变换 & 0.075 & 4.339 \\
方法 & 插值法 & 经验法变换 & -0.096 & 4.261 \\
& & 对数经验法 & -0.201 & 4.325 \\
\hline
\end{tabular}


$R M S E$ 总体最小的插值方法插值效果较好, 且 $R M S E$ 越小越好(孟庆香等, 2009)。由表 4 可知, 地 统计方法优于常规方法。地统计学中通常采用误 差均值 $(M E A N)$ 、均方根误差 $(R M S E)$ 、平均标准误差 $(A S E)$ 、标准平均误差 $(M S E)$ 和标准均方根误差 (RMSSE) 等 5 个指标评价预测精度。符合以下条件 则模型最优: 误差均值 $(M E A N)$ 的绝对值最接近于 0 、均方根误差 $(R M S E)$ 最小、平均标准误差 $(A S E)$ 最 接近于均方根误差 $(R M S E)$ 、标准均方根误差 $(R M S$ $S E$ )最接近于 1(易湘生等, 2012)。通过广义交叉验 证, 从表 5 可以看出,无变换的贝叶斯克里金插值结 果相对较好。

(3) 医疗设施空间可达性的综合评价

杨浦区医疗设施空间可达性插值分析可视化 结果如图 5 所示。从整体来看, 医疗设施对各街道 (镇)的服务不太均衡,具体分析如下：

1) 中部区域部分街道(镇)的医疗设施规模和数 量具有明显优势, 但存在同等级医院邻近布置、医 疗资源相对集中的情况,如大桥街道和五角场镇。 其中,深色环状高值区域主要集中在定海路街道、 大桥街道, 表明这 2 个区域具有较为明显的就医优 势。大桥街道拥有 2 家三级医院、1 家二级医院和 1 个社区卫生服务中心, 形成了较为完整的层级式就 医模式。而定海路街道拥有 1 家三级医院和 1 个社 区卫生中心, 也能为居民提供既高效又高质的医疗 服务。

当居住点周边配置完整层级体系的医疗设施、 居民就医的不同需求能得到较大满足时,居民就医 的总体空间可达性较好。对于未来医疗设施的布 局与规划,不仅要考虑新建医疗设施的服务规模, 更要考虑已有医疗设施的分布, 以便形成良好的层 级式就医模式。同时应避免同等级医院距离过近、 而部分区域医疗设施空白的情况,尽可能减少重复 建设和资源圥余。此外,为实现供需平衡,应将可

表 5 不同克里金插值方法检验比较

Tab.5 Comparison of the results calculated by different

Kriging interpolation methods

\begin{tabular}{lccc}
\hline \multirow{2}{*}{ 广义交叉验证 } & \multicolumn{3}{c}{ 经验贝叶斯克里金插值法 } \\
\cline { 2 - 4 } & 无变换 & 经验法 & 对数经验法 \\
\hline MEAN & 0.075 & -0.096 & -0.201 \\
$R M S E$ & 4.339 & 4.261 & 4.325 \\
$M S E$ & 0.023 & -0.026 & -0.020 \\
$R M S S E$ & 0.944 & 1.078 & 1.195 \\
$A S E$ & 4.197 & 4.031 & 3.217 \\
\hline
\end{tabular}

达性高值区域医疗服务能力向低值区域疏导和延 伸, 使建成区内居民的医疗设施可达性趋于均衡, 以满足服务的公平性。

2) 地铁 8 号线途经杨浦区内四平路街道、江浦 路街道、控江路街道、延吉新村街道、长白新村街 道、五角场镇、殷行街道部分区域,将附近的各级医 院有效地串联在一起,因此沿线附近的居民就医空 间可达性较好。说明地铁等交通条件的改善提高 了医疗设施的通达性,通过轨道交通和地面交通配 合能节省通行时间,为居民提供更多机会获取医疗 资源,并提高医疗设施及卫生资源的利用率。在国 家大力推行“城市公共交通优先”的背景下,优化公 共交通换乘线路、加强不同公共交通方式的系统联 系,弥补盲区,将大大提高医疗设施的可达性。

3) 杨浦区北部区域部分居民点数量较少且分 散、位置稍偏远，属于具有一定发展积淀的城市新 区, 区域内的市政基础设施建设已较为完善,但医 疗机构和公共交通服务还未达到与城市开发及基 础设施建设相匹配的水平, 居民往往需到附近街道 (镇)就医, 因此空间可达性相对较差。但随着区域 内人口逐渐聚集, 医疗设施逐步配备齐全, 加之地 铁 10 号线二期工程从新江湾城站北端延伸,服务杨 浦区北部地区，区域内居民的医疗设施空间可达性 也会随之提升。

插值分析可以由点及面地描述各居民点周围 区域的医疗设施空间可达性,但要了解全区整体医 疗设施空间可达性的空间分异特点还需借助空间

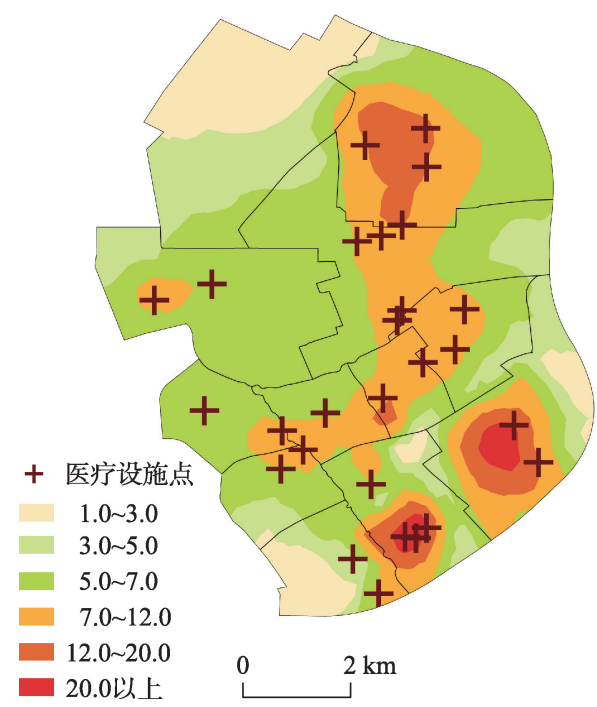

图 5 杨浦区医疗设施空间可达性插值分析

Fig.5 Interpolation analysis of spatial accessibility of medical facilities in Yangpu District 
冷热点 $\left(\right.$ Getis-Ord $\left.G_{i}^{*}\right)$ 分析, 以刻画出高一高聚类 (热点)和低一低聚类(冷点)的分布情况, 结果如图 6 所示。由图可知, 全区约 $2 / 3$ 的区域聚类不明显, 约 $1 / 3$ 的区域居民的医疗设施空间可达性分异明显。 全区可达性水平可以分为 3 类: $a$. 热点地区集中在 大桥街道、定海路街道以及殷行街道部分区域, 具有 医疗服务水平显著偏高的特点, 属于高水平聚类区 域; b. 平凉路街道及新江湾城街道部分居民点是冷 点地区, 属于医疗服务水平亟待改善的低水平聚类 区域, 需要通过加快医疗设施建设提高医疗服务供 给能力; $c$. 其余区域属于热点向冷点过渡的区域, 医 疗服务情况介于前两者之间, 改善次序排在冷点地 区之后。

\section{4) 不同等级医疗设施空间可达性分析}

为了更直观地显示不同等级医疗设施的空间 可达性差异, 根据各居民点的医疗设施空间可达性 值与全部居民点的医疗设施空间可达性的平均值 的比值, 将居民点的医疗设施空间可达性分为 5 个 等级, 分别为:差、较差、一般、较好、好(表 6)。从表

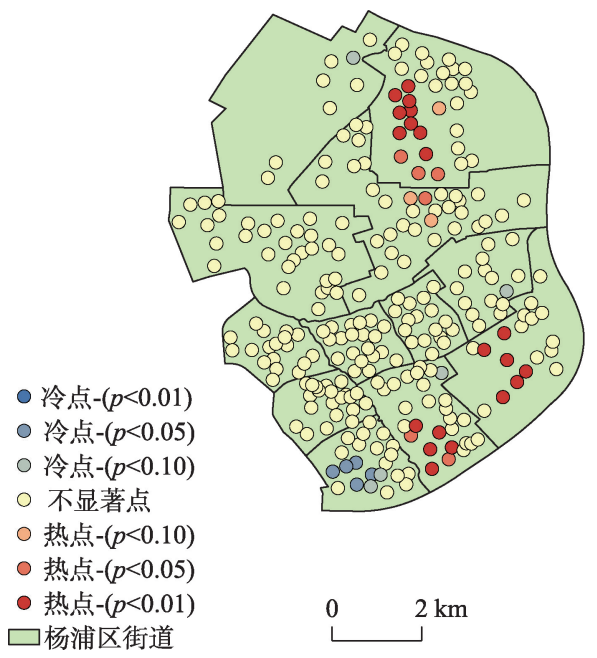

图 6 杨浦区医疗设施空间可达性 Getis-Ord $G_{i}^{*}$ 分析

Fig.6 Getis-Ord $G_{i}^{*}$ analysis of spatial accessibility of medical facilities in Yangpu District

表 6 医院分级模式下的居民点医疗设施空间可达性 情况对比

Tab.6 Spatial accessibility of medical facilities of different grades

\begin{tabular}{cccccc}
\hline 医院等级 & $\begin{array}{c}\text { 差 } \\
(<0.50)\end{array}$ & $\begin{array}{c}\text { 较差 } \\
(0.50 \sim 0.75)\end{array}$ & $\begin{array}{c}\text { 一般 } \\
(0.75 ~ 1.00)\end{array}$ & $\begin{array}{c}\text { 较好 } \\
(1.00 \sim 1.25)\end{array}$ & $\begin{array}{c}\text { 好 } \\
(>1.25)\end{array}$ \\
\hline 三级 $/ \%$ & 7.17 & 29.81 & 26.79 & 17.74 & 18.49 \\
二级 $\%$ & 64.91 & 9.43 & 4.91 & 3.02 & 17.74 \\
一级 $\%$ & 75.85 & 3.77 & 2.64 & 1.13 & 16.60 \\
\hline
\end{tabular}

中可以看出:一级医院 “差” 等级的占比为 $75.85 \%$, 说明超过 3/4 的居民点的医疗设施空间可达性远远 低于全区的平均值,而 “好”等级的占比为 $16.60 \%, 2$ 个等级的累积比超过 $90 \%$, 说明各居民点一级医院 可达性表现出明显的不均衡现象;二级医院相对于 一级医院而言, 居民点的医疗设施空间可达性的内 部差异性减小; 三级医院 5 个等级的占比较为均衡。

当 $\beta=1.8$ 时,各街道(镇)不同等级医疗设施空 间可达性雷达图如图 7 所示。由图 7 可知, 各街道 (镇)的三级医院空间可达性大多优于二级医院和一 级医院, 是因为三级医院设施规模和服务供给数量 均优于二级、一级医院, 且居民选择三级医院就医 的极限出行时间取值也大于二级医院和一级医 院。在各街道(镇)中, 大桥街道和控江路街道的三 级医院空间可达性最好, 定海路街道的二级医院空 间可达性较好, 各街道(镇)一级医院可达性普遍较 低。由于三级医院的服务范围广、对象多, 资源总 量远高于其他等级医院,再进行硬件条件改善的空 间相对较小。因此,在交通条件一定时,增加二级、 一级医院的数量, 依托三级医院的资源优势, 组建 医疗团队,促进优质资源纵向延伸,提升二级、一级 医院的设施规模和水平能更为有效地改善区域内 居民的医疗设施空间可达性。

\section{4 结论与讨论}

本文引人人口规模因子和医疗设施等级规模 影响系数改进潜能模型, 基于 GIS 空间分析技术, 以上海杨浦区为例对其医疗设施空间可达性进行 研究。改进的潜能模型综合考虑了居民点人口数 量、医疗设施服务能力、医疗设施与居民点之间的 出行阻抗、医疗设施不同等级规模对居民就医选择 的影响,能更为科学地刻画医疗设施空间可达性,

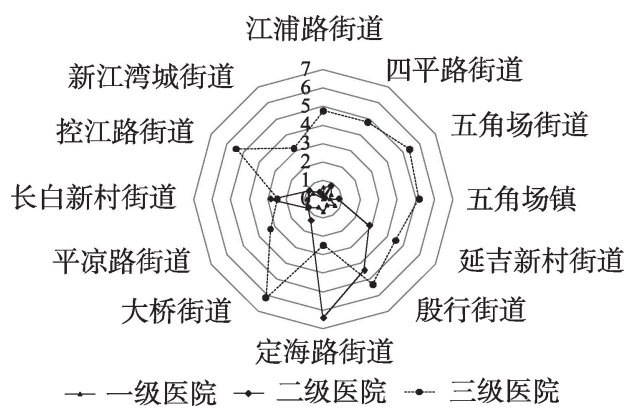

图 7 杨浦区各街道(镇)不同等级医疗设施空间可达性雷达图

Fig.7 Spatial accessibility of medical facilities in different subdistricts of Yangpu District 
准确度量区域内居民实际所能获得的医疗资源。 同时, 本文在运用 GIS 分析技术时, 针对上海道路 交通特点, 综合考虑了路面交通与轨道交通, 以居 住区为研究单元, 从而更加贴近实际。

研究结果表明: (1)从区域来看, 杨浦区中部区 域部分街道的医疗设施空间可达性相对较好, 北部 区域部分居民的医疗设施空间可达性相对较弱, (2) 从居民点来看, 大桥街道、定海路街道以及殷行街 道部分居民点医疗设施空间可达性较好, 但大桥街 道和五角场镇街道存在同等级医院临近配置、医疗 资源相对集中情况; 平凉路街道及新江湾城街道部 分居民点医疗设施空间可达性有待改善; (3从医疗 设施等级来看, 三级医院空间可达性整体优于二 级、一级医院, 大桥街道和控江路街道的三级医院 空间可达性最好, 定海路街道的二级医院空间可达 性较好。

本文的研究结果有助于了解杨浦区医疗设施 可达性状况, 研究方法可为其他类别的公共服务设 施或其他城市医疗设施的合理规划和布局提供借 鉴。然而, 本文还存在以下不足和有待进一步研究 之处: 一是由于受限于数据可得性, 本文所选的人 口数据只涉及人口总数, 未根据人口构成特征进行 分类, 而居民对医疗设施的需求不仅与人口数量有 关, 还与人口的构成有很大关系, 因此, 今后的研究 可纳人人口构成因素, 使分析结果更有针对性; 二 是在表征居民点位置时, 由于所获得的居住区面图 层数据有限, 无法以居住区面图层数据的几何质心 为人口重心, 而是选择以居住区的行政中心(居委 会)的地理坐标代表研究单元的人口重心。虽然通 过计算发现误差在可接受范围内, 但是在后续研究 中还有待搜集更完整的图层数据并细化研究单元, 使得计算结果更为准确合理, 找到缺医的最基层单 元; 三是针对不同等级规模的医疗设施的居民极限 出行时间及出行阻抗的设定还存在一定的主观性, 后续研究可利用不同的时间阈值进行空间可达性 分布的敏感性分析, 同时通过居民就医出行的大数 据回归分析得到研究区域实际的出行阻抗值。此 外,本文主要关注区域医疗设施空间可达性测算, 还有待在现有医疗设施空间可达性评价的基础上 进一步开展相应的配置优化研究。

\section{参考文献(References)}

陈晨, 修春亮. 2014. 基于交通网络中心性的长春市大型综 合医院空间可达性研究 [J]. 人文地理, 29(5): 81-87.
[Chen C, Xiu C L. 2014. Spatial accessibility of large general hospitals in Changchun City center based on street network centrality[J]. Human Geography, 29(5): 81-87.]

邓丽, 邵景安, 郭跃, 等. 2015. 基于改进的两步移动搜索法 的山区医疗服务空间可达性: 以重庆市石柱县为例 $[\mathrm{J}]$. 地理科学进展, 34(6): 716-725. [Deng L, Shao J A, Guo Y, et al. 2015. Spatial accessibility of medical services in mountainous regions based on modified two-step floating catchment area method: A case study of Shizhu County, Chongqing[J]. Progress in Geography, 34(6): 716-725.]

邓羽, 蔡建明, 杨振山, 等. 2012. 北京城区交通时间可达性 测度及其空间特征分析 [J]. 地理学报, 67(2): 169-178. [Deng Y, Cai J M, Yang Z S, et al. 2012. Measuring time accessibility with its spatial characteristics in urban areas of Beijing[J]. Acta Geographica Sinica, 67(2): 169-178.]

丁秋贤, 朱丽霞, 罗静. 2016. 武汉市养老设施空间可达性分 析 [J]. 人文地理, 31(2): 36-42. [Ding Q X, Zhu L X, Luo J. 2016. Analysing spatial accessibility to residential care facilities in Wuhan[J]. Human Geography, 31(2): 36-42.]

丁愫, 陈报章. 2017. 城市医疗设施空间分布合理性评估 [J]. 地球信息科学学报, 19(2): 185-196. [Ding S, Chen B Z. 2017. Rationality assessment of the spatial distributions of urban medical facility[J]. Journal of Geo-information Science, 19(2): 185-196.]

胡瑞山, 董锁成, 胡浩. 2012. 就医空间可达性分析的两步移 动搜索法: 以江苏省东海县为例 $[\mathrm{J}]$. 地理科学进展, 31 (12): 1600-1607. [Hu R S, Dong S C, Hu H. 2012. A Twostep Floating Catchment Area (2SFCA) method for measuring spatial accessibility to primary healthcare service in China: A case study of Donghai County in Jiangsu Province[J]. Progress in Geography, 31(12): 1600-1607.]

孔云峰, 李小建, 张雪峰. 2008. 农村中小学布局调整之空间 可达性分析: 以河南省巩义市初级中学为例 [J]. 遥感学 报, 12(5): 800-809. [Kong Y F, Li X J, Zhang X F. 2008. Analysis of spatial accessibility for school redistricting in rural China: A case study of the secondary schools in Gongyi City, Henan Province[J]. Journal of Remote Sensing, 12(5): 800-809.]

林康, 陆玉麒, 刘俊, 等. 2009. 基于可达性角度的公共产品 空间公平性的定量评价方法: 以江苏省仪征市为例 $[\mathrm{J}]$. 地理研究, 28(1): 215-224. [Lin K, Lu Y Q, Liu J, et al. 2009. Assessment of fair space of public product based on accessibility: A case of Yizheng City[J]. Geographical Research, 28(1): 215-224.]

孟庆香, 刘国涁, 杨勤科. 2009. 黄土高原年均温的空间插值 方法研究 [J]. 干旱区资源与环境, 23(3): 83-87. [Meng Q X, Liu G B, Yang Q K. 2009. Research on spatial interpolation methods of annual average temperature on Loess Plateau[J]. Journal of Arid Land Resources and Environment, 
23(3): 83-87.]

孟田田, 张晶. 2017. 北京城区就医可达性评价及空间特征 分析 [J]. 地理空间信息, 15(3): 62-65. [Meng T T, Zhang

J. 2017. Evaluation of health care service spatial accessibility and the analysis of the spatial distribution characteristics in Beijing[J]. Geospatial Information, 15(3): 62-65.]

宋正娜, 陈雯. 2009. 基于潜能模型的医疗设施空间可达性 评价方法 [J]. 地理科学进展, 28(6): 848-854. [Song Z N, Chen W. 2009. Measuring spatial accessibility to health care facilities based on potential model[J]. Progress in Geography, 28(6): 848-854.]

宋正娜, 陈雯, 车前进, 等. 2010. 基于改进潜能模型的就医 空间可达性度量和缺医地区判断: 以江苏省如东县为例 [J]. 地理科学, 30(2): 213-219. [Song Z N, Chen W, Che Q J, et al. 2010. Measurement of spatial accessibility to health care facilities and defining health professional shortage areas based on improved potential model: A case study of Rudong County in Jiangsu Province[J]. Scientia Geographica Sinica, 30(2): 213-219.]

孙瑜康, 吕斌, 赵勇健. 2015. 基于出行调查和 GIS 分析的县 域公共服务设施配置评价研究: 以德兴市医疗设施为例 [J]. 人文地理, 30(3): 103-110. [Sun Y K, Lv B, Zhao Y J. 2015. A study of county public service facilities distribution assessment based on behavior investigation and GIS: A case study of medical facilities in Dexing[J]. Human Geography, 30(3): 103-110.]

汤鹏飞, 向京京, 罗静, 等. 2017. 基于改进潜能模型的县域 小学空间可达性研究: 以湖北省仙桃市为例 [J]. 地理科 学进展, 36(6): 697-708. [Tang P F, Xiang J J, Luo J, et al. 2017. Spatial accessibility analysis of primary schools at the county level based on the improved potential model: A case study of Xiantao City, Hubei Province[J]. Progress in Geography, 36(6): 697-708.]

陶卓霖, 程杨, 戴特奇. 2014. 北京市养老设施空间可达性评 价[J]. 地理科学进展, 33(5): 616-624. [Tao Z L, Cheng Y, Dai T Q. 2014. Measuring spatial accessibility to residential care facilities in Beijing[J]. Progress in Geography, 33(5): 616-624.]

陶卓霖, 程杨, 戴特奇, 等. 2017. 公共服务设施空间可达性 评价中的参数敏感性分析 [J]. 现代城市研究, 32(3): 3035. [Tao Z L, Cheng Y, Dai T Q, et al. 2017. Sensitivity analysis of parameters in measuring spatial accessibility to public service facilities[J]. Modern Urban Research, 32(3): 30-35.]

佟圣楠, 陈航航. 2017. 基于街道尺度的医疗设施可达性评 价: 以深圳市为例 [J]. 卫生经济研究, (2): 31-34. [Tong S N, Chen H H. 2017. Evaluation of accessibility of medical facilities based on street scale: A case study in Shenzhen City[J]. Health Economics Research, (2): 31-34.]

吴建军, 孔云峰, 李斌. 2008. 基于 GIS 的农村医疗设施空间 可达性分析: 以河南省兰考县为例 $[\mathrm{J}]$. 人文地理, 23(5):
37-42. [Wu J J, Kong Y F, Li B. 2008. The spatial accessibility analysis of rural medical facilities based on GIS: A case study of Lankao County, Henan Province[J]. Human Geography, 23(5): 37-42.]

熊娟, 罗静, 彭菁, 等. 2012. 基于可达性的县域医疗服务均 等化分析: 以湖北省松滋市为例 [J]. 人文地理, 27(5): 2529, 119. [Xiong J, Luo J, Peng J, et al. 2012. Equalization analysis of medical service of county level based on accessibility: A case study of Songzi, Hubei Province[J]. Human Geography, 27(5): 25-29, 119.]

易湘生, 李国胜, 尹衍雨, 等. 2012. 土壤厚度的空间插值方 法比较: 以青海三江源地区为例 [J]. 地理研究, 31(10): 1793-1805. [Yi X S, Li G S, Yin Y Y, et al. 2012. Comparison on soil depth prediction among different spatial interpolation methods: A case study in the Three-River Headwaters Region of Qinghai Province[J]. Geographical Research, 31(10): 1793-1805.]

曾文, 向梨丽, 张小林. 2017. 南京市社区服务设施可达性的 空间格局与低收人社区空间剥夺研究 $[\mathrm{J}]$. 人文地理, 32 (1): 73-81. [Zeng W, Xiang L L, Zhang X L. 2017. Research in spatial pattern of accessibility to community service facilities and spatial deprivation of low income community in Nanjing[J]. Human Geography, 32(1): 73-81.]

钟少颖, 杨金金, 陈锐. 2016. 层级性公共服务设施空间可达性 研究: 以北京市综合性医疗设施为例 [J]. 地理研究, 35 (4): 731-744. [Zhong S Y, Yang X, Chen R. 2016. The accessibility measurement of hierarchy public service facilities based on multi-mode network dataset and the two-step 2SFCA: A case study of Beijing's medical facilities[J]. Geographical Research, 35(4): 731-744.]

Cheng G, Zeng X K, Duan L, et al. 2016. Spatial difference analysis for accessibility to high level hospitals based on travel time in Shenzhen, China[J]. Habitat International, 53: 485-494

Fan P L, Xu L H, Yue W Z, et al. 2017. Accessibility of public urban green space in an urban periphery: The case of Shanghai[J]. Landscape and Urban Planning, 165: 177-192.

Gharani P, Stewart K, Ryan G L. 2015. An enhanced approach for modeling spatial accessibility for in vitro fertilization services in the rural Midwestern United States[J]. Applied Geography, 64: 12-23.

Kalogirou S, Foley R. 2006. Health, place and Hanly: Modelling accessibility to hospitals in Ireland[J]. Irish Geography, 39(1): 52-68.

Ortega E, López E, Monzón A. 2012. Territorial cohesion impacts of high-speed rail at different planning levels[J]. Journal of Transport Geography, 24: 130-141.

Pan J, Zhao H Q, Wang X L, et al. 2016. Assessing spatial access to public and private hospitals in Sichuan, China: The 
influence of the private sector on the healthcare geography in China[J]. Social Science \& Medicine, 170: 35-45.

Peeters D, Thomas I. 2000. Distance predicting functions and applied location-allocation models[J]. Journal of Geographical Systems, 2(2): 167-184.

Porta S, Latora V, Wang F H, et al. 2012. Street centrality and the location of economic activities in Barcelona[J]. Urban Studies, 49(7): 1471-1488.

Reggiani A, Bucci P, Russo G. 2011. Accessibility and impedance forms: Empirical applications to the German commuting networks[J]. International Regional Science Review, 34 (2): 230-252.

Siegel M, Koller D, Vogt V, et al. 2016. Developing a composite index of spatial accessibility across different health care sectors: A German example[J]. Health Policy, 120(2): 205-212.

Suárez P, Mayor M, Cueto B. 2012. The accessibility to employment offices in the Spanish labour market[J]. Papers in Regional Science, 91(4): 823-848.

Weibull. J W. 1976. An axiomatic approach to the measurement of accessibility[J]. Regional Science and Urban Economics, 6(4): 357-379.

Wu C, Ye X Y, Du Q Y, et al. 2017. Spatial effects of accessibility to parks on housing prices in Shenzhen, China[J]. Habitat International, 63: 45-54.

Yang N, Chen S Y, Hu W L, et al. 2016. Spatial distribution balance analysis of hospitals in Wuhan[J]. International Journal of Environmental Research and Public Health, 13 (10): 971.

\title{
Spatial accessibility of urban medical facilities based on improved potential model: A case study of Yangpu District in Shanghai
}

\author{
CHENG Min*, LIAN Yuejiao \\ (School of Management, Shanghai University, Shanghai 200444, China)
}

\begin{abstract}
Medical services are the basic requirement of the public and are closely related to public health. The rationality of the layout and spatial accessibility of medical facilities are closely related to the right of the public to healthcare. Spatial accessibility of medical facilities represents the convenience for residents to obtain medical services. Using GIS and an improved potential model that introduces population scale factor and medical facility grade influence coefficient to traditional potential model, the spatial accessibility of urban medical facilities in Yangpu District of Shanghai was studied. By applying a spatial interpolation method, accessibility of medical facilities of Yangpu District in 2016 was obtained. Moreover, hotspot analysis was used for the cluster analysis of spatial accessibility. The results indicate that the spatial accessibility of urban medical facilities in different community of Yangpu District varies. The spatial accessibility in the central area of Yangpu District is higher than that of other areas. Spatial accessibility is relatively high in Daqiao subdistrict, Dinghai Road subdistrict, and some areas of Yinxing subdistrict. Hospitals of the same grade in the subdistricts of the central area are so closely located that medical resources are of high concentration in these areas. Overall, spatial accessibility of tertiary hospitals is better than secondary and primary hospitals in Yangpu. The improved potential model can evaluate the spatial accessibility of urban medical facilities reasonably by integrating population in demands, travel impedance between residential areas and medical facilities, and the influence of medical facility grade on residents' choice of medical services. By using GIS, the difference of spatial accessibility of urban medical facilities can be visualized. Furthermore, areas with insufficient medical resources can be defined. The results can not only enrich public facility spatial planning theory, but also provide references for the decision making of relevant government departments.
\end{abstract}

Key words: medical facilities; spatial accessibility; improved potential model; GIS; Yangpu District; Shanghai Municipality 